\title{
Food sources and inulin consumption in school-aged children
}

\author{
G. Morillo-Santander, L. Curtin, S. Williams, C. Edwards and A. L. Garcia \\ Human Nutrition, School of Medicine, Dentistry and Nursing, College of Medical, Veterinary and Life Sciences, \\ University of Glasgow, Glasgow, UK
}

Dietary fibre intake is below recommendations in children and some staple foods, rich in inulin, may help to increase its consumption. Inulin a prebiotic dietary fibre ${ }^{(1)}$ is mainly found in plant-based foods. Food reformulation has led to an increase on inulin content in various food products recently, yet there is little research on the level of inulin consumption from food sources in the diet especially in children. Aim: To explore the food sources and intake of inulin in school-aged children.

Children were recruited between the Summer and Fall of 2019 in purposely designed child interactive science activities. Diet intake was recorded using one 24-hour recall, and with the aid of food images parents helped their child to remember the foods eaten. For the assessment of inulin a list of foods was created using food composition data reporting on inulin-type fructan content ${ }^{(2)(3)}$. The amount of inulin from reported homemade and takeaway food was calculated using the mean ingredients of three recipes. Nutritics software was used to check for standard portions.

A total of 154 school-aged children, median age 7 years old, IQR (12-5) female/male ratio (61/39\%) were recruited from the least deprived areas (65.3\%) of Scotland. Median inulin consumption was $1.3 \mathrm{~g} / \mathrm{day}$, IQR (7.1-0). Grain and cereal products contributed over half the inulin intake (58.4\%). Within this group, bread accounted for over a third of inulin (36.5\%), followed by breakfast cereals and biscuits with only $13.3 \%$ and $5.1 \%$, respectively. The second highest contributing food group was takeaway and homemade dishes which accounted for $21.7 \%$ of inulin consumption, including vegetable dishes with $9 \%$. The vegetable, potatoes, beans and fruits food group contributed the least amount of inulin with $11.9 \%$ and $8 \%$, respectively.

In this population of school-aged children, the main food sources of inulin come from grain and cereal products and mixed meals from which carrots and onions are the likely contributing ingredients to inulin consumption. These data will help inform future intervention studies of increased dietary fibre consumption in children.

\section{References}

1. Roberfroid M, Gibson GR, Hoyles L, et al. (2010) Br J Nutr 104, S1-63.

2. Biesiekierski JR, Rosella O, Rose R, et al. (2011) J Hum Nutr Diet 24(2), 154-76.

3. Whelan K, Abrahmsohn O, David GJ, et al. (2011) Int J Food Sci Nutr 62(5), 498-503. 